\title{
A TWO-STAGE DUAL THRESHOLD AGENT-BASED MODEL OF INNOVATION ADOPTION
}

\author{
Dr. Arpan Y. Jani \\ University of Wisconsin - River Falls \\ Department of Computer Science and Information Systems \\ 410 S. 3rd Street, River Falls WI 54022 USA \\ arpan.jani@uwrf.edu
}

\begin{abstract}
Studies on diffusion of innovation have found that individuals and firms often do not recognize the benefits of an innovation in the early stages though they may eventually adopt it, which is reflected in the S-shaped diffusion curve. This could be attributed to information about an innovation spreading gradually. Even when ample information is available about an innovation, evaluation of information may be biased in favour of maintaining status quo. This paper presents an agent-based model which focuses on biased evaluation of information. It is assumed that agents have dual thresholds for evaluating competing information: one for maintaining the status quo choice and the other for switching to the alternative. Simulation experiments were conducted by varying the evaluation thresholds and the time frame over which the information was evaluated to investigate its influence on the adoption of an innovation. Results match the S-shaped diffusion curve under certain conditions.
\end{abstract}

Keywords: Diffusion of Innovation, Agent-based Modelling, Cognitive Biases

\section{INTRODUCTION}

Even though a new innovation may have clear benefits, it may take a long time for it to be adopted widely. Rogers' theory of diffusion of innovation (Rogers, 1962) identifies five categories of adopters: innovators, early adopters, early majority, late majority, and laggards. Innovators tend to be the first adopters whereas laggards tend to be among the last to adopt a new innovation. Geroski (2000) discusses alternate models of technology diffusion: the epidemic model, the probit model, population ecology based models, and the information cascade model. In the epidemic model it is assumed that the information spreads slowly over time and due to this the adoption rate differs across individuals (or firms) (Geroski, 2000). Probit models consider individual characteristics such as the switching costs or opportunity costs (Geroski, 2000) faced by individuals in adopting a new innovation. Information cascades perspective (Geroski, 2000) suggests that the early adopters of an innovation may go through a serious evaluation of the alternatives available before making a choice. Subsequent adopters may take a cognitive shortcut and be more likely to choose the same alternative instead of going through the extensive cognitive processing (e.g. see Bikhchandani et al., 1992).

While epidemiological models pose that diffusion of innovation is limited by the availability of information, studies in behavioral decision making have shown that even if all the information is available, how this information is evaluated may influence the choices made by individuals. Individuals may exhibit a confirmation bias (Nickerson, 1998) where they seek out information that supports their existing position and avoid information that could contradict their position. From this perspective, once individuals have made a choice, they may be more likely to seek information that justifies this choice even if other information may suggest that a better option is available. Individuals may also exhibit a status quo bias (Samuelson and Zickhauser, 1988) so that they may be more likely to stick to a chosen alternative even when a better choice is available. 
Agent-based models incorporate simple decision rules while recognizing that agents make decisions based on the local context e.g. what information is available to them. This paper develops an agent-based model of diffusion of innovation where the focus is not just on availability of information itself but also how this information is evaluated. While gradually more information may be available about an innovation, biased processing of this information may lead to delays in adoption of the innovation. The model can also incorporate heterogeneity among agents in how carefully they evaluate the information and the varying time frame over which the information is gathered and evaluated.

\section{A TWO-STAGE DUAL THRESHOLD MODEL OF DECISION MAKING}

Threshold models have been used in social and behavioural sciences to explain social behaviour (Granovetter, 1978; Schelling, 1971). In psychology, threshold-based model of decision making have been proposed where individuals are assumed to make a choice once a threshold is reached (Curley, et al., 2018) which provides support for the choice. While a single threshold is simpler to consider in the context of decision making, it may be better to incorporate two thresholds in situations where the decision is to switch to an alternative: whether to maintain or reject the existing choice and to accept the alternate. It is as if individuals maintain dual standards for evaluation of the alternatives: one for the choice already made and the other for switching to a new alternative. This way one can consider the case where individuals may have a lower threshold for maintaining the current choice but may have a higher threshold for switching to an alternative.

Figure 1 presents a two-stage, dual threshold model of decision making. It assumes that an agent may have an initial belief i.e. benefits of product A, which would justify the initial choice made. This belief would have been formed based on the information that was available about product $\mathrm{A}$. However, suppose a new innovation, product $\mathrm{B}$, is also available in the market for which gradually more information becomes available. It is assumed that individuals have two thresholds, $\boldsymbol{\theta}_{\boldsymbol{I}}$ and $\boldsymbol{\theta}_{2}$. $\boldsymbol{\theta}_{\boldsymbol{I}}\left(0<=\boldsymbol{\theta}_{\boldsymbol{I}}<=1\right)$ is the threshold of evidence needed for maintaining one's current belief (i.e. product $\mathrm{A}$ is the best option available and one should continue to adopt it) and $\boldsymbol{\theta}_{2}\left(0<=\boldsymbol{\theta}_{2}<=1\right)$ is the threshold of evidence required to change to the alternate belief (i.e. product $\mathrm{B}$ is superior and should be adopted). Individuals gather information about the products which provides varying evidence in support of both alternatives. Thus, at any given time, there is relative evidence $\emptyset_{1}(0<=$ $\left.\emptyset_{1}<=1\right)$ that supports the adoption of product A and relative evidence $\emptyset_{2}\left(0<=\emptyset_{2}<=1\right)$ that supports the adoption of product $\mathrm{B}$.

Agents first compare the evidence $\boldsymbol{\emptyset} \boldsymbol{1}$ with threshold $\boldsymbol{\theta} \boldsymbol{1}$ and if the accumulated evidence clears this threshold, the agents do not need to engage in further information processing and would maintain their current belief (to continue to adopt Product A). This reflects the well-known confirmation bias. However, if there isn't sufficient evidence to support the initial belief i.e. the first threshold is not met, agents would look at the evidence for the alternative. If the evidence for the alternative is not sufficient, the agents will continue to hold the initial belief, thus reflecting the status-quo bias. If the relative evidence for the alternative is sufficient i.e. $\boldsymbol{\emptyset}_{2}$ is higher than the threshold $\boldsymbol{\theta}_{2}$, the agent will be ready to change its belief. This concludes the first stage which may result in either the agents maintaining their current beliefs or becoming ready to change as doubts have appeared.

In the second stage, the agents who are ready to change their belief again look for evidence to support their initial beliefs and if there is sufficient relative evidence i.e. $\emptyset 1$ greater than the threshold $\boldsymbol{\theta 1}$, the agent would go back to holding their initial belief. However, if the evidence is not sufficient for their initial belief, the agent will compare the relative evidence $\boldsymbol{\emptyset} \mathbf{2}$ with threshold $\boldsymbol{\theta} \mathbf{2}$. If the evidence clears the threshold, then the agent will change to the new belief (i.e. product B has superior benefits and should be adopted). If the evidence does not clear the threshold, then the agent would continue to be in the "ready to change" state. Hence, the two-stage dual threshold model represents the resistance to change that individuals have when considering adoption of a new alternative. 


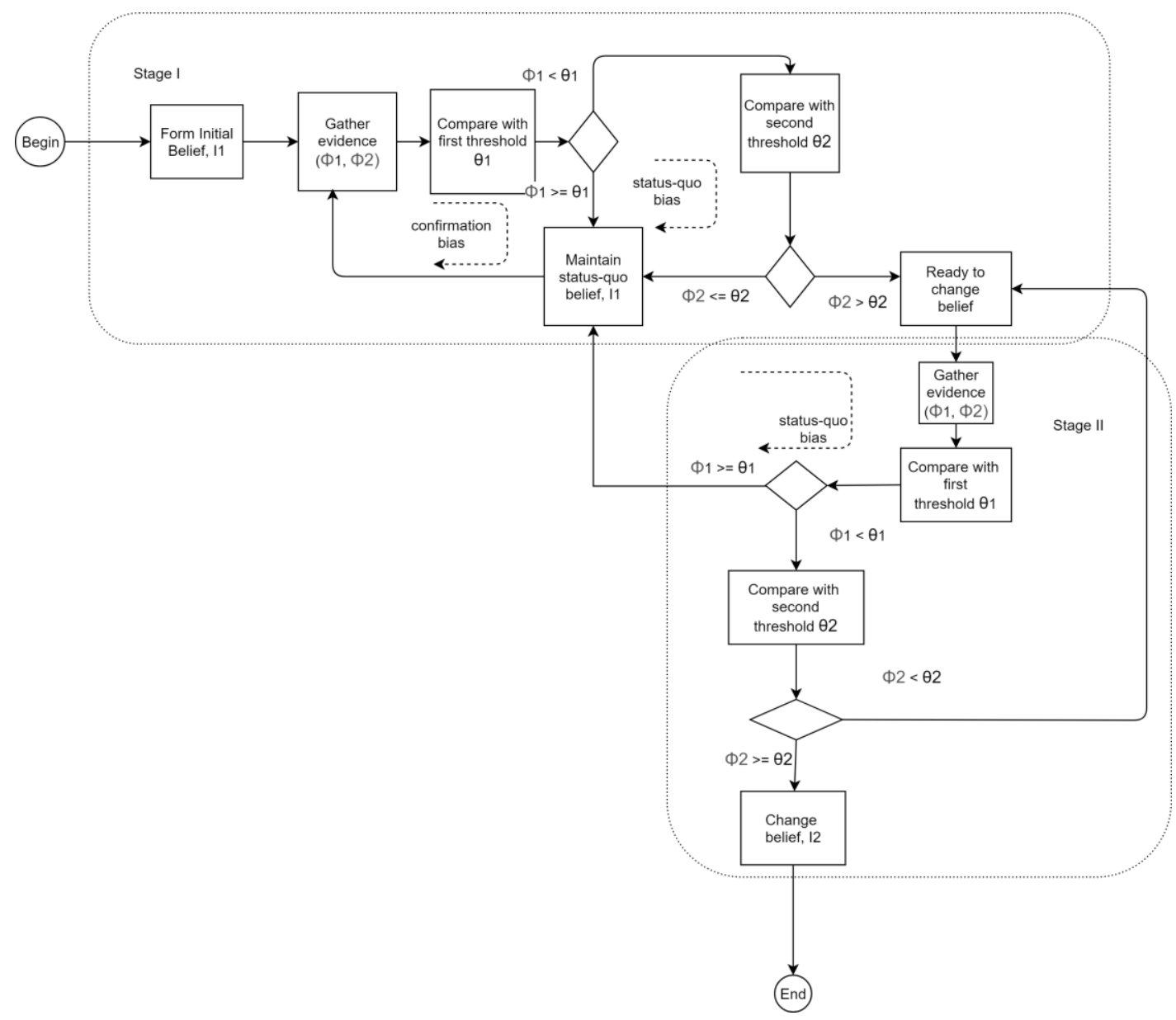

Figure 1 Two-stage dual threshold model of belief change

\subsection{An Agent-based Model of Innovation Diffusion}

An agent-based model of innovation diffusion that incorporates the two-stage dual threshold model of belief revision is presented here. The model was implemented in NetLogo (Wilensky, 1999). A torus grid with $100 \times 100$ size which wrapped both horizontally and vertically was used. This represented 10201 patches, with each patch representing a piece of information. The patch could be of one of two colours. The patch colour represented a piece of information that either supported belief A (e.g. Product A should be adopted) or belief B (e.g. product B should be adopted). A green patch represented information in support of product $\mathrm{A}$ and a yellow patch represented information in support of product B. Initially the entire grid was initialized to be green which indicated that all evidence was in support of product A (the current dominant technology). As product B, the new innovation, is introduced into the market, slowly more information becomes available. In each time step, one patch turned from green to yellow indicating that new information about product $\mathrm{B}$ was available. Each piece of information had credibility $\mathrm{C}$ that varied from 0 to 1 , drawn from a uniform distribution. Hence, coming across information with higher credibility will lead to greater support for a particular belief.

$\boldsymbol{C 1}$ is the credibility of a piece of information about initial belief (i.e. product A should be adopted) and $\boldsymbol{C} 2$ is the credibility of a piece of information about alternate belief (i.e. product $\mathrm{B}$ should be adopted).

$$
\begin{aligned}
& 0 \leq \boldsymbol{C} \boldsymbol{1} \leq 1 \text { where } \boldsymbol{C} \boldsymbol{1} \sim \text { uniform }(0,1) \\
& 0 \leq \boldsymbol{C} \boldsymbol{2} \leq 1 \text { where } \boldsymbol{C} \boldsymbol{2} \sim \text { uniform }(0,1)
\end{aligned}
$$


The relative evidence $\emptyset_{2}$ in support of the alternative grows over time since more information is available about the benefits of product B whereas relative evidence $\emptyset_{1}$ decreases over time as new information crowds out the existing information.

\subsubsection{Model Initialization}

2000 agents were created and were assumed to have an initial belief supporting adoption of product A that they had currently adopted product A which was the dominant technology. These agents randomly moved around the grid and collected evidence in the form of information pieces with a certain credibility. The number of patches visited represented the information pieces that the agent came across, each with a certain degree of credibility. In the beginning of the simulation since most patches were green, the agents would only come across information supporting their current choice and hardly any that would support switching to the alternative. However, in each time step, one of the green patches changed to yellow indicating that over time the information supporting adoption of the alternative increased linearly whereas the information supporting the continued adoption of the default choice linearly decreased. Agents evaluated the collected evidence in a decision time frame that could range from 10 time steps to 100 time steps.

Let's say that during decision time frame $t$, an agent $i$ has collected $m$ pieces of evidence in support of the initial belief (i.e. continue to use product A) and $n$ pieces of evidence in support of the alternate belief (adopt new product B). $\boldsymbol{\emptyset}_{1_{i t}}$ is the ratio of the sum total of the evidence collected in time frame $\boldsymbol{t}$ by agent $\boldsymbol{i}$ that is in favour of initial belief to the sum total of all evidence collected by agent $\boldsymbol{i}$ and $\boldsymbol{\emptyset} \boldsymbol{2}_{i t}$ is the ratio of sum total of the evidence collected in time frame $t$ by agent $\boldsymbol{i}$ that is in favour of alternate belief to the sum total of evidence collected by agent $i$.

Proportion of evidence that supports initial belief

$$
\emptyset 1_{i t}=\left(\sum_{j=1}^{n} C 1_{j}\right) /\left(\sum_{j=1}^{n} C 1_{j}+\sum_{k=1}^{m} C 2_{k}\right)
$$

Proportion of evidence that supports alternate belief

$$
\emptyset 2_{i t}=\left(\sum_{k=1}^{m} C 2_{k}\right) /\left(\sum_{j=1}^{n} C 1_{j}+\sum_{k=1}^{m} C 2_{k}\right)
$$

Each agent has evidence thresholds $\boldsymbol{\theta}_{1}$ and $\boldsymbol{\theta}_{2}$ for evaluating the evidence for the initial belief and alternate belief respectively. As discussed earlier, if there is weak relative evidence to support the initial belief but there is strong relative evidence to support the alternate belief, the agent will change to the state "ready to change belief." If there is a continued lack of support for the initial belief but strong support for the alternate belief, the agent will switch to the new belief.

\subsubsection{Simulation Runs}

The BehaviourSpace feature of NetLogo enables running of simulation experiments multiple times while changing the values of its parameters across these runs. The NetLogo simulation had the following three parameters: $\boldsymbol{\theta}_{1}, \boldsymbol{\theta}_{2}$, and Decision Time Frame. Table 1 shows the range of values that were varied across multiple simulation runs. The total runs were 250 . 
Table 1 Parameter Values - Simulation Run

\begin{tabular}{|c|c|c|}
\hline Parameter & Range of Values & Number of values \\
\hline Threshold 1 $(\boldsymbol{\theta 1})$ & $0.1-0.9$ increments of 0.2 & 5 \\
\hline Threshold 2 $(\boldsymbol{\theta} 2)$ & $0.1-0.9$ increments of 0.2 & 5 \\
\hline $\begin{array}{c}\text { Decision Time } \\
\text { Frame (Ticks) }\end{array}$ & $10-100$ increments of 10 & 10 \\
\hline \multicolumn{2}{|c|}{ Total Simulation Runs } & $5 * 5 * 10=250$ \\
\hline
\end{tabular}

\subsection{Results and Discussion}

Data analysis was conducted using RStudio and R. For data visualization, ggplot2 package (Wickham, 2016) was used. Figure 2 shows the rate of adoption under varying evaluation threshold values and decision time frames. Each line chart in the panel of charts below shows the adoption rate for the combination of $\boldsymbol{\theta 1}$ (Threshold 1) and $\boldsymbol{\theta} \mathbf{2}$ (Threshold 2) values. The rows show $\boldsymbol{\theta 1}$ value varying from 0.1 to 0.9 in increments of 0.2 and the columns show $\boldsymbol{\theta 2}$ values varying from 0.1 to 0.9 in increments of 0.2. Each line chart also shows the adoption rate for various decision time frames from 10 time steps (ticks) to 100 time steps (ticks). Many of the line charts match the classic Sshaped diffusion curve for specific combinations of $\boldsymbol{\theta 1}, \boldsymbol{\theta} \mathbf{2}$ and decision time frames.

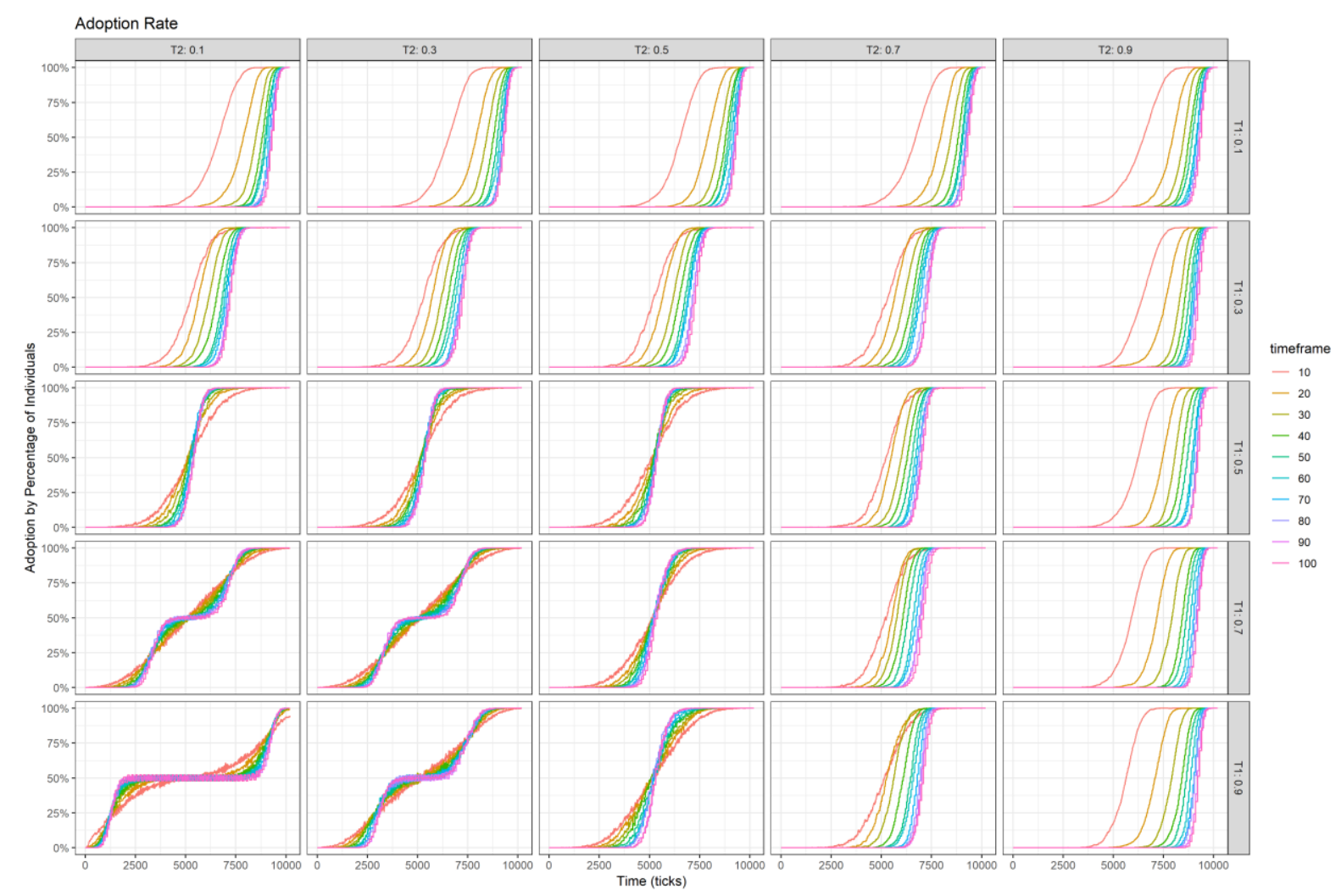

Figure 2 Adoption Rates With Varying Evaluation Thresholds and Decision Time Frames

Figure 3 shows the adoption at midway point of the simulation where rows show $\boldsymbol{\theta} \boldsymbol{1}$ value varying from 0.1 to 0.9 and columns show $\boldsymbol{\theta 2}$ values varying from 0.1 to 0.9 . Each vertical column/bar shows the adoption rate for a given decision time frame. The decision time frame varies from 10 ticks to 100 ticks. 


\section{Jani}

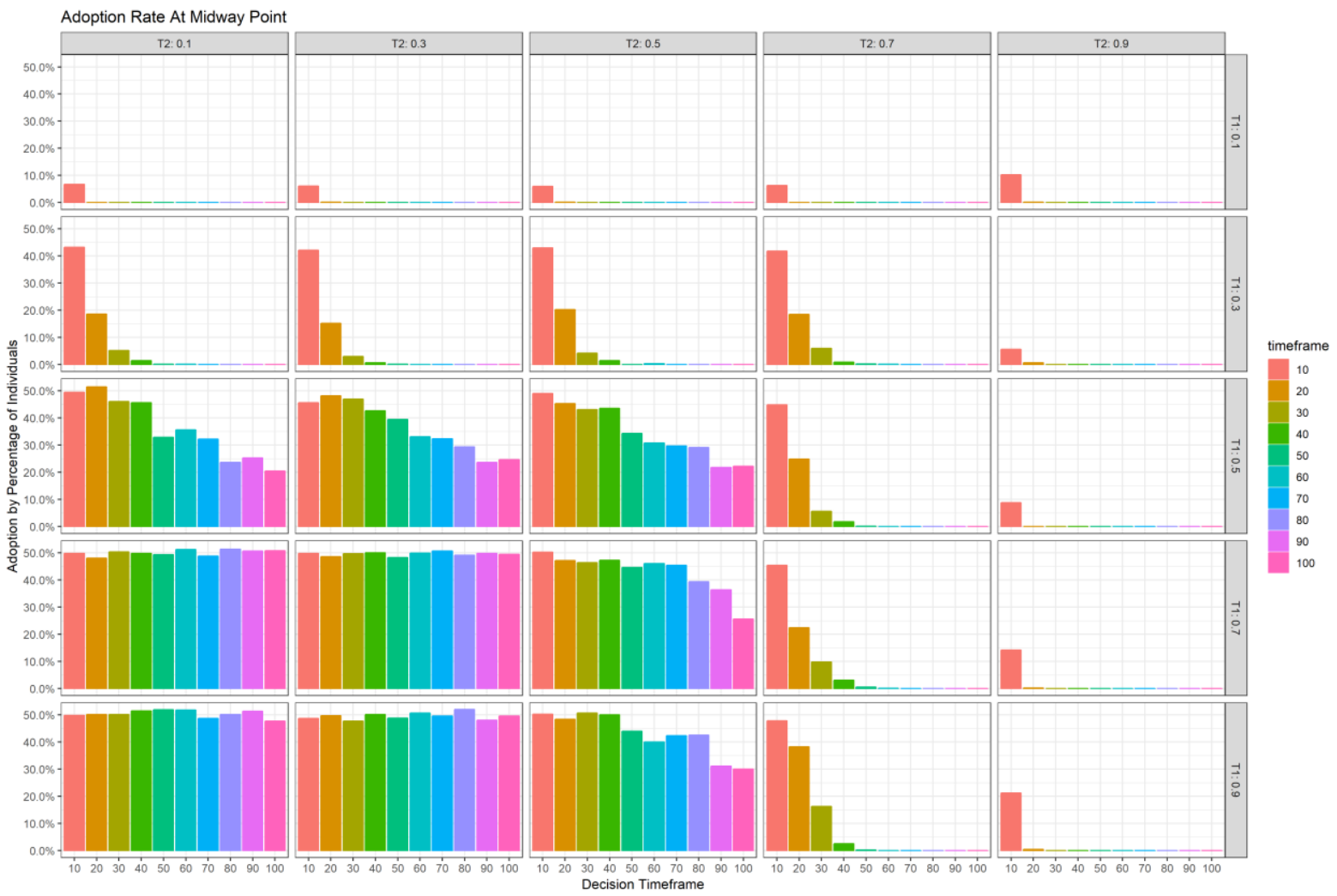

Figure 3 Adoption percentage at mid-point of the simulation run

In both figures 2 and 3 above, one can observe that as the decision time frame increases from 10 time steps (ticks) to 100 time steps (ticks), the adoption gets increasingly delayed and the transition to the alternative occurs more steeply.

As the first row of the panel in Figure 3 shows, when $\boldsymbol{\theta 1}$ value is low $(\boldsymbol{\theta} \boldsymbol{1}=0.1)$, the adoption is quite slow irrespective of the value of $\boldsymbol{\theta 2}$. This is because the standard of evidence needed to support the default choice is quite low. When both $\boldsymbol{\theta 1}$ and $\boldsymbol{\theta} 2$ are in the middle range $(\boldsymbol{\theta 1}=0.5, \boldsymbol{\theta} 2=0.5)$, adoption rate is the highest with the lowest decision time frame but decreases as the decision time frame increases. As seen in the lower right hand panel of Figure 3, when $\boldsymbol{\theta 1}$ is high (which means that the agents would need a very high evidence in order to continue with the default choice), and $\boldsymbol{\theta 2}$ is low, the decision time frame is not too influential. The lower right hand corner represents high values for both $\boldsymbol{\theta 1}$ and $\boldsymbol{\theta 2}$, which means that one requires a very high evidence standard to either continue with the default choice or to switch. In this case, the adoption rate is also slow.

Figure 4 shows the overall behavior of the model. Even though the available information about an innovation increases linearly over time, the outcome i.e. adoption of this innovation unfolds in a nonlinear manner. The shape of the adoption curve is influenced by the two threshold values and the decision time frame.

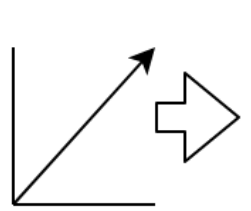

Information Availability

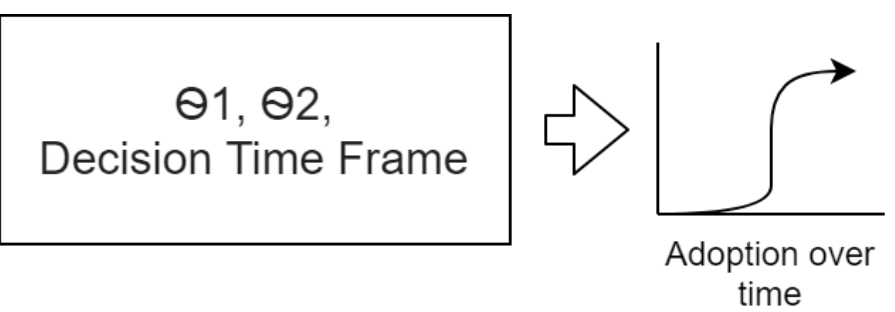

Figure 4 Input, Output and Key Model Parameters 


\section{CONCLUSION}

An agent-based model of innovation diffusion is presented here that focuses on the biased evaluation of information available about an innovation. Compared to other models of diffusion of innovation, this is primarily a cognitive, information-processing model. It is assumed that greater evidence in support of the adoption of the innovation is available over time. Biased evaluation of this evidence influences the adoption pattern. The model shows that when there is low amount of information available about the innovation, there is not much adoption but once more information is available, under certain conditions, the adoption rate exceeds what the objective evidence may suggest. That is, everyone in the population adopts the new innovation in the later period as if complete evidence was in its favour. Thus, the adoption rate either lags or leads the availability of information.

\section{REFERENCES}

Bikhchandani S, Hirshleifer D and Welch I (1992). A theory of fads, fashion, custom, and cultural change in informational cascades. Journal of Political Economy 100(5):992-1026.

Curley L J, Maclean R, Murray J and Laybourn P (2018). Decision science: A new hope. Psychological Reports 122(6): 2417-2439.

Granovetter M (1978). Threshold models of collective behavior. American Journal of Sociology 83(6): 1420-1443.

Nickerson R S (1998). Confirmation bias: A ubiquitous phenomenon in many guises. Review of General Psychology 2(2): 175-220.

Rogers E M (1962). Diffusion of Innovations. Glencoe: Free Press.

R Core Team (2017). R: A language and environment for statistical computing. R Foundation for Statistical Computing, Vienna, Austria. URL https://www.R-project.org/.

RStudio Team (2015). RStudio: Integrated Development for R. RStudio, Inc., Boston, MA URL http://www.rstudio.com/.

Samuelson W and Zeckhauser R (1988). Status quo bias in decision making. Journal of Risk and Uncertainty, 1: 7-59.

Wickham, H (2016). ggplot2: Elegant Graphics for Data Analysis. New York: Springer-Verlag.

Wilensky, U (1999). NetLogo. http://ccl.northwestern.edu/netlogo/.

\section{AUTHOR BIOGRAPHIES}

Arpan Jani received his $\mathrm{PhD}$ in Business Administration from the University of Minnesota in 2005. $\mathrm{He}$ is currently an Associate Professor in the Department of Computer Science and Information Systems at the University of Wisconsin - River Falls. His research interests include agent-based modelling, information systems and decision support, behavioural ethics, and judgment \& decision making under conditions of risk and uncertainty. 